\title{
Structure and properties of flax vs. lyocell fiber-reinforced polylactide stereocomplex composites
}

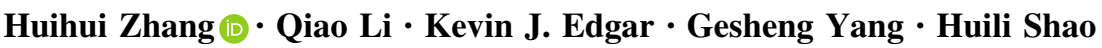

Received: 8 December 2020 / Accepted: 22 July 2021

(C) The Author(s), under exclusive licence to Springer Nature B.V. 2021

\begin{abstract}
A commonly used natural cellulose fiber (flax) and a regenerated cellulose fiber (Lyocell) were used at $20 \mathrm{wt} \%$ to reinforce polylactide stereocomplex (sc-PLA) composites. Composites were prepared by melt compounding cellulose fibers and an equivalent proportion of PLLA/PDLA, followed by injection molding. The structures and properties of these two kinds of cellulose fiber/sc-PLA composites were compared and evaluated. The results showed that the total crystallinity and stereocomplex crystallite content of composites could be increased by reinforcing with cellulose fibers, and Lyocell fibers were more effective in accelerating crystallinity and the formation of stereocomplex crystallites than flax fibers. Mechanical properties of Lyocell fibers were much poorer than those of flax fibers, and the interfacial adhesion values of Lyocell/sc-PLA composites were inferior to those of flax/sc-PLA composites. Lyocell/
\end{abstract}

H. Zhang $(\bowtie) \cdot$ Q. Li · G. Yang · H. Shao

State Key Laboratory for Modification of Chemical Fibers and Polymer Materials, College of Material Science and Engineering, Donghua University, Shanghai 201620, China

e-mail: hhzhang@dhu.edu.cn

\section{K. J. Edgar}

Macromolecules Innovation Institute, Virginia Tech, Blacksburg, VA 24061, USA

\section{K. J. Edgar}

Department of Sustainable Biomaterials, Virginia Tech, Blacksburg, VA 24061, USA
sc-PLA composites showed higher impact strength and similar tensile strength vs. flax/sc-PLA composites, but the Young's modulus values of Lyocell/scPLA composites were lower than those of flax/sc-PLA composites. The Vicat softening temperatures of both flax/sc-PLA and Lyocell/sc-PLA composites were increased to nearly $100{ }^{\circ} \mathrm{C}$ higher than that of PLLA. Lyocell/sc-PLA composites showed the highest Vicat softening temperature of $\sim 170{ }^{\circ} \mathrm{C}$.

Keywords Polylactide stereocomplex (sc-PLA) . Flax $\cdot$ Lyocell $\cdot$ Composites

\section{Introduction}

Poly (lactic acid) (PLA) is a biodegradable and biobased thermoplastic polyester with high modulus and strength, and it is considered to be one prominent alternative for the replacement of conventional petroleum-based plastics. However, application of PLA is still restricted because of its brittleness and low heat distortion temperature (HDT) (Ghosh et al. 2007). Therefore, recently researchers have focused on improving the thermal stability and impact strength of PLA-based materials (Jin et al. 2019; Liu and Zhang 2011; Mokhena et al. 2018).

In order to improve the mechanical properties of PLA while retaining its biodegradability, some natural 
cellulose fibers, such as wood fibers (Huda et al. 2006), bamboo fibers (Tokoro et al. 2007), kenaf (Huda et al. 2008; Ochi 2008), jute (Arao et al. 2015; Ma and Joo 2011), hemp (Hu and Lim 2007; Sawpan et al. 2011), sisal (Orue et al. 2015), and flax (Aydin et al. 2011; Nassiopoulos and Njuguna 2015), have been used to reinforce PLA bio-composites. In addition, use of regenerated cellulose fibers (typically Rayon (viscose fiber) and Lyocell fibers) to reinforce PLA has been demonstrated by several groups (Bax and Müssig 2008; Ganster and Fink 2006; Graupner et al. 2009; Yu et al. 2019). These natural or regenerated cellulose fiber-reinforced PLA composites showed improved mechanical properties, in particular improved impact resistance vs. neat PLA. However, PLA thermal stability cannot be effectively improved by reinforcing with cellulose fibers.

Since Ikada first reported stereocomplex formation between enantiomeric poly (L-lactide) (PLLA) and poly (D-lactide) (PDLA) (Ikada et al. 1987), stereocomplexation between these optical isomers has become one of the most effective methods to improve PLA thermal resistance, because stereocomplexed polylactide (sc-PLA) shows a melting temperature (ca. $230{ }^{\circ} \mathrm{C}$ ), ca. $50{ }^{\circ} \mathrm{C}$ higher than the melting temperatures of the homo-crystallized polylactides (PLLA or PDLA). The reason for the high melting temperature of sc-PLA has been proposed to be the strong methylcarbonyl van der Waals interactions in the PLLA/ PDLA stereocomplex, much stronger than equivalent interactions in homocrystalline PLA with its differently arrange unit cell (Brizzolara et al. 1996; Zhang et al. 2005). The so-called $\beta$-form of the stereocomplex crystallites is formed by packing monomers of opposite absolute configuration alternatingly side by side, that is a D-monomer unit alternating with an L-monomer unit (Brizzolara et al. 1996).

Studies have confirmed that stereoselective association between D- and L-polymers occurred preferably when PDLA and PLLA were blended in equivalent proportions (1:1) (Tsuji and Ikada 1993, 1999). However, homo-crystallites (hc) and stereocomplex crystallites (sc) were reported to coexist, although sc crystallites predominated, in sc-PLA manufactured by melt blending equivalent proportions of PLLA/PDLA, followed by conventional compression or injection molding (Li et al. 2018; Srithep et al. 2015). The obtained sc-PLA showed inferior mechanical properties to those of PLLA or PDLA, likely due to degree of polymerization (DP) loss caused by the high temperature during the melting process (Xu et al. 2012).

Therefore, we hypothesized that combining stereocomplex formation with reinforcement by cellulose fibers would increase simultaneously the heat-resistance and mechanical properties of PLA, while retaining biodegradability due to the biodegradable nature of cellulose fibers. We have reported flax/scPLA bio-composites with enhanced heat resistance and improved mechanical properties (Ming et al. 2017; Zhang et al. 2015, 2020). Tensile strength (54\%), Young's modulus (132\%), and notched Izod impact strength (343\%) of these flax/sc-PLA composites each were substantially improved in comparison with sc-PLA properties. The Vicat softening temperature of the $30 \%$ flax/sc-PLA composite exceeded $162{ }^{\circ} \mathrm{C}$, or nearly $100{ }^{\circ} \mathrm{C}$ higher than that of PLLA.

Each different cellulose fiber has its own advantages. For example, the natural plant fibers (such as flax or ramie) have high values of specific strength and specific modulus, while regenerated cellulose fibers with their controllable shapes and diameters therefore show adjustable and controllable properties, good uniformity, and elongation values that often exceed those of natural fibers. Therefore, we hypothesize that sc-PLA composite characteristics can be varied and controlled by reinforcing with different natural or regenerated cellulose fibers, and we further hypothesize that these composites will have properties that are of value for different technical applications.

In this work, a typical natural cellulose fiber (flax) and an important regenerated cellulose fiber (Lyocell) were selected as reinforcing agents. The selected fiber and an equivalent proportion of PLLA/PDLA were melt compounded, followed by injection molding to prepare sc-PLA composites containing $20 \mathrm{wt} \%$ of the cellulose fiber. We evaluate herein the structure and properties of these two kinds of cellulose fiber/sc-PLA composite.

\section{Experimental}

Materials

PLLA and PDLA in pellet form were supplied by Nature Works LLC, USA and Purac Corbion Biochem., Singapore, respectively. Important 
Table 1 Characteristics of PLLA and PDLA pellets

\begin{tabular}{llll}
\hline Samples & $\mathrm{M}_{\mathrm{v}}\left(\times 10^{4} \mathrm{~g} / \mathrm{mol}\right)^{\mathrm{a}}$ & $\mathrm{T}_{\mathrm{g}}\left({ }^{\circ} \mathrm{C}\right)^{\mathrm{b}}$ & $\mathrm{T}_{\mathrm{m}}\left({ }^{\circ} \mathrm{C}\right)^{\mathrm{b}}$ \\
\hline PLLA & 3.7 & 66.2 & 169.4 \\
PDLA & 9.6 & 65.5 & 193.7 \\
\hline
\end{tabular}

${ }^{\mathrm{a}} \mathrm{M}_{\mathrm{v}}$ was calculated according to the Mark-Houwink equation: $[\eta]=5.45 \times 10^{-4} \mathrm{M}_{\mathrm{v}}{ }^{0.73}$

${ }^{\mathrm{b}} \mathrm{T}_{\mathrm{g}}$ and $\mathrm{T}_{\mathrm{m}}$ were measured by DSC

characteristics of the PLLA and PDLA used are listed in Table 1.

Flax fibers (scutched flax) were supplied by Harbin Yangguang Flax Textile Co., Ltd., China. Lyocell fibers with length $38 \mathrm{~mm}$ were provided by Lenzing AG, Austria.

Characterization of cellulose fibers

\section{Fourier transform infrared spectroscopy (FTIR)}

FTIR spectra of cellulose fibers were measured on a Nicolet 6700 spectrometer (Thermo Fisher Scientific Inc., USA) using compression molded film samples at a range of $400-4000 \mathrm{~cm}^{-1}$.

\section{Scanning electron microscopy (SEM)}

Surfaces of different cellulose fibers were observed using Quanta 250 environment scanning electron microscopes (ESEM, FEI Hongkong Co. Ltd, China) at $10 \mathrm{kV}$ acceleration voltage.

\section{Mechanical properties measurement}

Mechanical properties of different cellulose fibers were measured with an XD-1 Fiber Fineness Tester (Donghua University, Shanghai, China) and an XQ1A Tensile Tester (Donghua University, Shanghai, China). The statistical results came from 20 measurements for each specimen.

\section{Thermogravimetric analysis (TGA)}

TGA was performed on a TG 209 F1 Thermogravimetric Analyzer (Netzsch Co., Germany) in the range of temperature from 30 to $600{ }^{\circ} \mathrm{C}$ under a nitrogen atmosphere. The scan rate was maintained at $20{ }^{\circ} \mathrm{C} /$ $\min$.

Preparation of cellulose fiber/ sc-PLA composites

Before compounding, flax or Lyocell fibers were cut into $4 \mathrm{~mm}$ lengths, then the short fibers, PLLA, and PDLA pellets were pre-dried in a vacuum oven at $60{ }^{\circ} \mathrm{C}$ for $12 \mathrm{~h}$. Then PLLA and PDLA pellets (1:1 wt: wt) and enough fiber to comprise $20 \%$ by weight of the mixture were compounded using a Rheocord 90 torque rheometer (Haake Co., Germany) with a rotating rate of $50 \mathrm{rpm}$ at $220{ }^{\circ} \mathrm{C}$ for $5 \mathrm{~min}$ for uniform mixing of fiber and polymer matrix. Then the compound was cut into small pieces and then injection molded using a SZ-5-C injection molding machine (Dehong Rubber and Plastic Machinery Co. Ltd., China) to prepare two kinds of sample bars: rectangle bars (for tensile test) and the dog bone shaped bars (for impact test). The injection and mold temperatures were $225{ }^{\circ} \mathrm{C}$ and $55^{\circ} \mathrm{C}$, respectively. The injection pressure was $0.4 \mathrm{MPa}$ and the dwell time was $10 \mathrm{~s}$.

For comparison, control samples of pure PLLA and sc-PLA (PLLA/PDLA = 1:1) were also prepared by this method, whereas PLLA was processed at the temperature of $190{ }^{\circ} \mathrm{C}$.

Characterization of cellulose fiber/sc-PLA composites

\section{Differential scanning calorimetry (DSC)}

DSC measurements were performed on a Modulated DSC 2910 (TA Instruments Ltd, USA). The sample was subjected to a single heating scan from $40{ }^{\circ} \mathrm{C}$ to $240{ }^{\circ} \mathrm{C}$ at a heating rate of $10{ }^{\circ} \mathrm{C} / \mathrm{min}$ under a $\mathrm{N}_{2}$ flow rate of $40 \mathrm{~mL} / \mathrm{min}$. A very thin slice of sample (8-10 mg) was taken from a cross section at the center of injection molded tensile bars. The glass transition temperatures $\left(T_{g}\right)$, cold crystallization temperatures $\left(T_{c c}\right)$, and melting temperatures $\left(T_{m, h c}, T_{m, s c}\right)$ of cellulose fiber/sc-PLA composites, as well as the enthalpy of homo-crystal $\left(\Delta H_{h c}\right)$ and stereocomplex crystal $\left(\Delta H_{s c}\right)$, were determined from the single heating DSC scan.

The total degree of crystallinity $\left(X_{c}\right)$ of each cellulose fiber/sc-PLA composite was calculated by using the following equation (Sarasua et al. 2005; Zhang et al. 2020): 
$X_{\mathrm{c}}(\%)=\frac{\Delta H_{m, h c}+\Delta H_{m, s c}-\Delta H_{c c}}{w \times \Delta H_{m(\text { blend })}^{0}} \times 100 \%$

where, $\Delta H_{m, h c}$ and $\Delta H_{m, s c}$ are the experimental melting enthalpies of the $\alpha$ form of homopolymer crystals and the $\beta$ form of the stereocomplex crystals, respectively. $\Delta H_{c c}$ is the cold crystallization enthalpy, and $w$ is the weight fraction of PLA in the composites. $\Delta H_{m \text { (blend) }}^{0}$ is the theoretical value of the melting enthalpy for perfect crystals, which can be calculated as follows:

$\Delta H_{m(\text { blend })}^{0}=\Delta H_{m, h c}^{0} \times f_{h c}+\Delta H_{m, s c}^{0} \times f s c$

where $\Delta H_{m, h c}^{0}$ and $\Delta H_{m, s c}^{0}$ are the theoretical enthalpy values for the polylactide $\alpha$ form homopolymer crystallites $(93.6 \mathrm{~J} / \mathrm{g}$ ) and the $\beta$ stereocomplex crystallites $(142 \mathrm{~J} / \mathrm{g})$, respectively. $f_{h c}$ and $f_{s c}$ are the relative amounts of the homo- and stereocomplex crystallites, respectively, developed during processing under nonisothermal conditions, and can be calculated as follows:

$f_{h c}(\%)=\frac{\Delta H_{m, h c}}{\Delta H_{m, h c}+\Delta H_{m, s c}} \times 100 \%$

$f_{s c}(\%)=\frac{\Delta H_{m, s c}}{\Delta H_{m, h c}+\Delta H_{m, s c}} \times 100 \%$

The crystallinity of stereocomplex crystallites $\left(\mathrm{X}_{\mathrm{sc}}\right)$ was obtained from the following equation:

$X_{\mathrm{sc}}(\%)=X_{c} \times f_{s c} \times 100 \%$

By contrast, the crystallinity degree of injection molded PLLA was calculated according to the following equation:

$X_{\mathrm{c}}(\%)=\frac{\Delta H_{m, h c}-\Delta H_{c c}}{\Delta H_{m, h c}^{0}} \times 100 \%$

\section{Thermogravimetric analysis (TGA)}

TGA was performed on a TG 209 F1 Thermogravimetric Analyzer (Netzsch Co., Germany) in the range of temperature from 30 to $600{ }^{\circ} \mathrm{C}$ under a nitrogen atmosphere. The scan rate was maintained at $20^{\circ} \mathrm{C} /$ $\min$.

\section{Mechanical properties testing}

Tensile properties of cellulose fibers/sc-PLA composites were tested by using an Instron 5969 universal testing machine (Instron Co., USA) at $2 \mathrm{~mm} / \mathrm{min}$. The notched Izod impact strengths of composites were determined by using a XJJUD-50Q Izod Pendulum impact tester (Chengde COTS Scientific Instruments, Co. Ltd., China) with pendulum energy of $1 \mathrm{~J}$. All mechanical property results were reported as averages from five specimens for each sample.

\section{Water contact angle measurement}

A contact angle analyzer (Attension Theta, Biolin Scientific, Finland) was used to measure the contact angle of the probe liquids (water, $3 \mu \mathrm{L}$ ) in air on the surface of rectangle sample bars. For each sample a minimum of three different readings were recorded.

\section{Scanning electron microscopy (SEM)}

The fracture surfaces created by sample tensile tests were observed using a Quanta 250 environment scanning electron microscope (ESEM, FEI Hongkong, China). All specimens were sputter coated with a thin layer of gold prior to examination.

Vicat softening temperature (VST)

VST was measured according to the ASTM D1525-07 by using an XWB-300E Thermal Deformation \& Vicat Softening Temperature Tester (Chengde Kecheng Testing Machine Co. Ltd, China). The sample was tested with a load of $1 \mathrm{~kg}$ and a heating rate of $120^{\circ} \mathrm{C} / \mathrm{h}$.

\section{Results and discussions}

Comparison of structure and mechanical properties of cellulose fibers

\section{FTIR analysis}

Figure 1 shows the FTIR spectra of flax and Lyocell fibers. The peak at $2918 \mathrm{~cm}^{-1}$ of flax fibers was more intense and sharper than that of Lyocell fibers, and there was a peak at $2851 \mathrm{~cm}^{-1}$, which was not present 


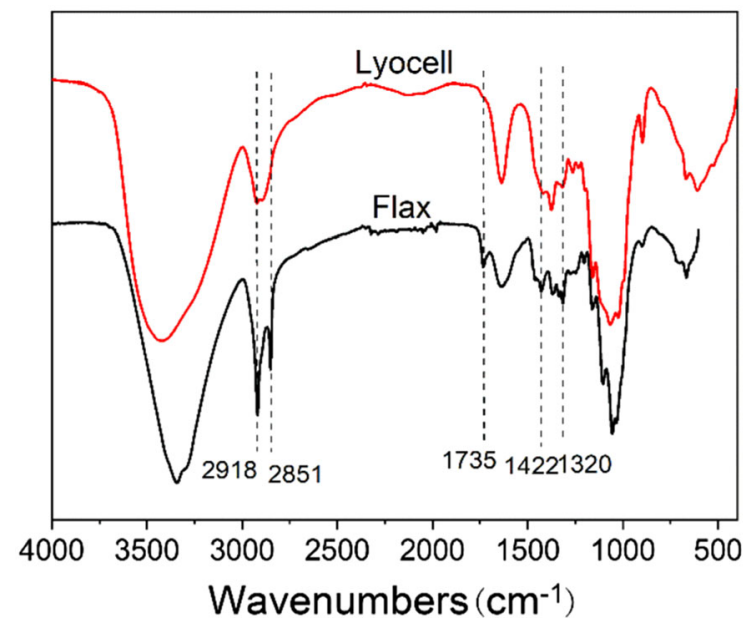

Fig. 1 FTIR spectra of flax and Lyocell fibers

in Lyocell fibers, indicating there was hemicellulose in flax fibers, because the peaks at $2918 \mathrm{~cm}^{-1}$ and $2851 \mathrm{~cm}^{-1}$ were attributed to $\mathrm{C}-\mathrm{H}$ stretching vibration of $\mathrm{CH}_{2}$ and $\mathrm{CH}$ groups in cellulose, and hemicellulose (Mwaikambo and Ansell 2002; Zhang et al. 2015). Moreover, the presence of a peak at $1735 \mathrm{~cm}^{-1}$ associated with the carbonyl $(\mathrm{C}=\mathrm{O})$ stretching of acetyl groups was notable, which further confirmed the presence of hemicellulose in the flax fibers (Biagiotti et al. 2004; Sgriccia et al. 2008). In addition, absorbances at $1422 \mathrm{~cm}^{-1}$ and $1320 \mathrm{~cm}^{-1}$, corresponding to the aromatic skeletal vibrations and syringyl ring breathing with $\mathrm{C}-\mathrm{O}$ stretching in lignin (Río et al. 2007), were observed in flax fibers, but were not obvious in Lyocell fibers (prepared from dissolving pulp and therefore containing little or no lignin (Jiang et al. 2020)).

It can be concluded based on the above FTIR analysis that hemicellulose and lignin existed in flax fibers because the scutched flax fibers in this study were not degummed; while the Lyocell regenerated cellulose fibers contained essentially no lignin, and hemicellulose, as expected since they are prepared from purified dissolving pulp.

\section{SEM analysis}

Figure 2 shows SEM images of flax and Lyocell fiber surfaces. Surfaces of flax fibers were not as smooth as those of regenerated Lyocell fibers. The flax fibers were present in bundles of elementary cellulose fibers likely held together mainly by lignin and hemicellulose, based on FTIR results. In contrast, Lyocell fibers exhibited smooth surfaces without any obvious crevices or flaws. These differences in cellulose fiber surface morphology may influence the properties of their composites.

\section{Mechanical properties analysis}

The mechanical properties of flax and Lyocell fibers are listed in Table 2. It is evident from the fiber fineness data that flax fibers were thicker than Lyocell fibers, which is consistent with the SEM images (Fig. 2). Moreover, tensile strength and initial modulus of flax fibers were significantly higher than those of Lyocell fibers, whereas Lyocell fibers exhibited much higher elongation than flax fibers, as described by others (Graupner et al. 2014). It is worth noting that high variability was found for the mechanical properties of flax due to the natural nonuniformity of flax fibers, but Lyocell fibers showed relatively low variability with regard to mechanical properties.

\section{Thermal performance analysis}

Figure 3 shows TG curves of flax and Lyocell fibers. It can be noticed that Lyocell fibers exhibited a little higher onset degradation temperature than flax due to the existence of hemicellulose and lignin in flax. It was reported that lignin began to degrade from $160{ }^{\circ} \mathrm{C}$, the pyrolysis of hemicellulose mainly happened at 220-315 ${ }^{\circ} \mathrm{C}$, which were lower than that of cellulose at $315-400{ }^{\circ} \mathrm{C}$ (Yang et al. 2007). Therefore, the pyrolysis of flax fibers occurred quickly than Lyocell fibers. However, the thermal degradation rate of flax was lower than Lyocell fibers at higher temperature, and more residue weight was obtained for flax at $600{ }^{\circ} \mathrm{C}$, which may be caused by the lignin in flax. Lignin was more difficult to decompose, as its weight loss happened in a wide temperature range (from 160 to $900{ }^{\circ} \mathrm{C}$ ) and the generated very high solid residue ( 40 wt.\%) (Yang et al. 2007). On the whole, the thermal stability difference of flax and Lyocell fibers may have no influence on the properties of cellulose fibers/sc-PLA composites because those fibers are thermal stable at the processing temperature of composites $\left(220-225^{\circ} \mathrm{C}\right)$. 

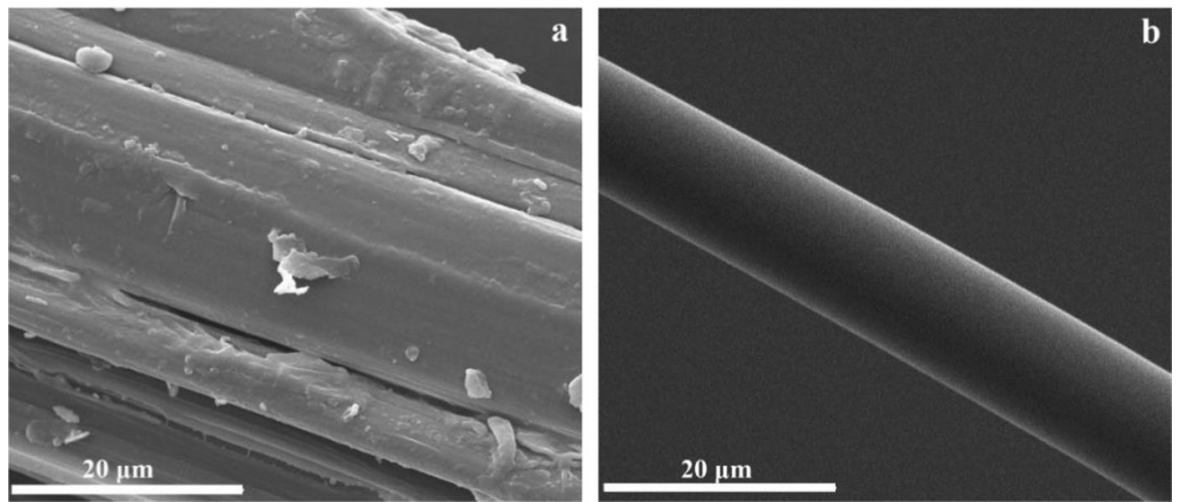

Fig. 2 SEM images of surface of flax (a) and Lyocell fibers (b)

Table 2 Mechanical properties of flax and Lyocell fibers

\begin{tabular}{llllr}
\hline Samples & $\begin{array}{l}\text { Fineness } \\
(\text { dtex })\end{array}$ & $\begin{array}{l}\text { Tensile strength } \\
(\mathrm{cN} / \text { dtex })\end{array}$ & $\begin{array}{l}\text { Initial modulus } \\
(\mathrm{cN} / \mathrm{dtex})\end{array}$ & $\begin{array}{l}\text { Elongation } \\
(\%)\end{array}$ \\
\hline Flax & $2.67 \pm 0.48$ & $7.12 \pm 3.62$ & $317.4 \pm 105.4$ & $3.4 \pm 2.4$ \\
Lyocell & $1.43 \pm 0.06$ & $3.47 \pm 0.19$ & $39.8 \pm 3.8$ & $15.3 \pm 1.8$ \\
\hline
\end{tabular}

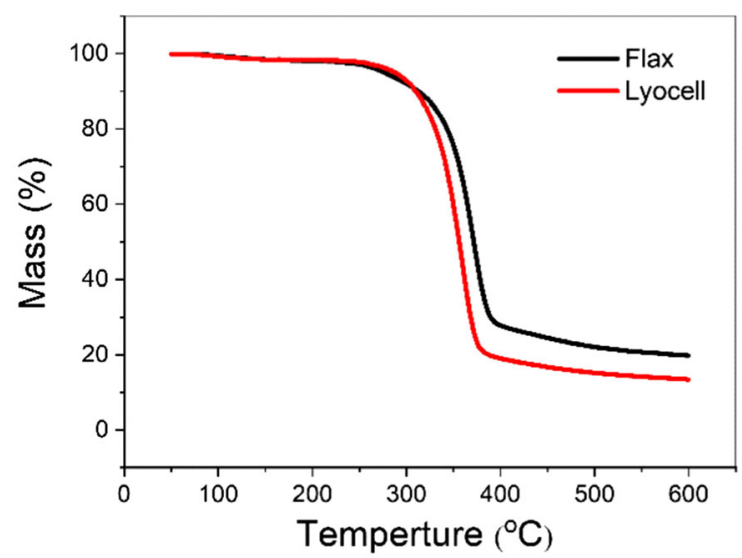

Fig. 3 TG curves of flax and Lyocell fibers

Comparison of structure and properties of different cellulose fiber/sc-PLA composites

\section{DSC analysis}

Figure 4 shows DSC heating scan curves of injectionmolded cellulose fiber/sc-PLA composites, and those of PLLA and sc-PLA (PLLA/PDLA = 1:1 blend) injection molded controls. The detailed thermal parameters derived from the DSC curves are listed in

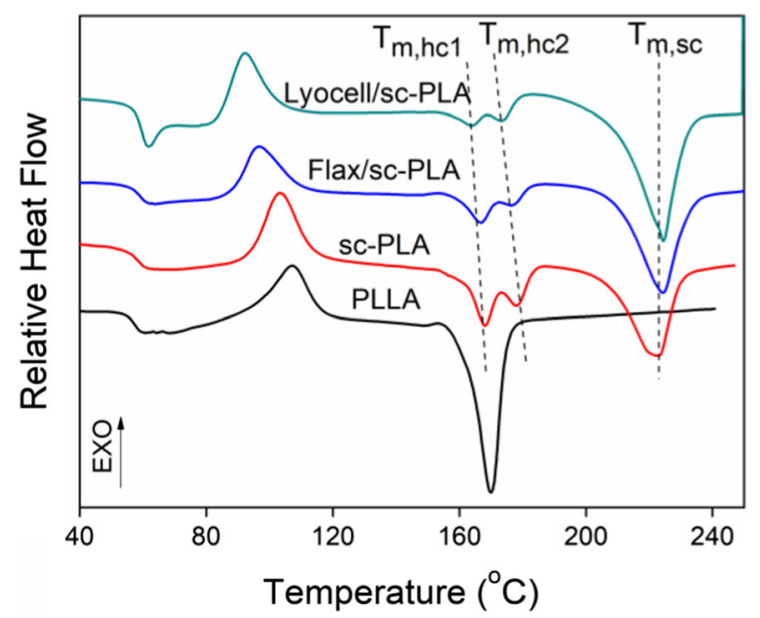

Fig. 4 DSC curves of injection-molded PLLA, sc-PLA and different cellulose fiber/sc-PLA composites

Table 3. It can be seen that injection- molded PLLA exhibited only one melting peak at $164.1{ }^{\circ} \mathrm{C}$, attributed to homocrystallites (hc), whereas sc-PLA and the two cellulose fibers/sc-PLA composites (based on Lyocell and flax) showed multiple melting peaks at 160-180 ${ }^{\circ} \mathrm{C}$ and $222-225^{\circ} \mathrm{C}$, which were assigned to melting of hc and stereocomplex crystallites (sc), respectively. That is to say, sc crystallites were formed 
Table 3 DSC data of injection-molded PLLA, sc-PLA and cellulose fiber/sc-PLA composites

\begin{tabular}{|c|c|c|c|c|c|c|c|c|c|c|}
\hline Samples & $\begin{array}{l}\mathrm{T}_{\mathrm{g}} \\
\left({ }^{\circ} \mathrm{C}\right)\end{array}$ & $\begin{array}{l}\mathrm{T}_{\mathrm{cc}} \\
\left({ }^{\circ} \mathrm{C}\right)\end{array}$ & $\begin{array}{l}\mathrm{T}_{\mathrm{m}, \mathrm{hc}} \\
\left({ }^{\circ} \mathrm{C}\right)\end{array}$ & $\begin{array}{l}\mathrm{T}_{\mathrm{m}, \mathrm{sc}} \\
\left({ }^{\circ} \mathrm{C}\right)\end{array}$ & $\begin{array}{l}\Delta \mathrm{H}_{\mathrm{cc}} \\
(\mathrm{J} / \mathrm{g})\end{array}$ & $\begin{array}{l}\Delta \mathrm{H}_{\mathrm{m}, \mathrm{hc}} \\
(\mathrm{J} / \mathrm{g})\end{array}$ & $\begin{array}{l}\Delta \mathrm{H}_{\mathrm{m}, \mathrm{sc}} \\
(\mathrm{J} / \mathrm{g})\end{array}$ & $\begin{array}{l}f_{\mathrm{sc}} \\
(\%)\end{array}$ & $\begin{array}{l}X_{\mathrm{sc}} \\
(\%)\end{array}$ & $\begin{array}{l}X_{\mathrm{c}} \\
(\%)\end{array}$ \\
\hline PLLA & 58.9 & 106.5 & 164.1 & - & 31.26 & 41.97 & - & - & - & 8.2 \\
\hline sc-PLA & 58.4 & 103.2 & $168.1 / 178.5$ & 222.2 & 26.99 & 20.02 & 36.12 & 64.3 & 15.1 & 23.4 \\
\hline Flax/sc-PLA & 59.0 & 96.5 & $166.8 / 177.3$ & 224.4 & 17.58 & 11.03 & 39.4 & 79.1 & 24.4 & 30.9 \\
\hline Lyocell/sc-PLA & 59.4 & 92.3 & $163.6 / 174.2$ & 224.4 & 21.47 & 8.4 & 49.48 & 85.5 & 28.8 & 33.7 \\
\hline
\end{tabular}

predominantly in all cellulose fibers/sc-PLA composites, although a small amount of hc crystallites coexisted. The coexistence of hc and sc is reasonable because both stereocomplexation and homocrystallization run parallel in a competitive manner during cooling of sc-PLA, as confirmed by many reports about sc-PLA prepared by melt blending (Ming et al. 2017; Xu et al. 2012).

The two cellulose fiber/sc-PLA composites showed higher stereocomplex melting temperatures and lower homocomplex melting temperatures, especially notable in the Lyocell/sc-PLA composites, indicating the existence of more perfect sc and less perfect hc in composites, which may be related to fiber surface topography (Mathew et al. 2006).

The crystallinity $\left(\mathrm{X}_{\mathrm{c}}\right.$, Table 3$)$ of PLLA was only $8.2 \%$ due to the fast cooling rate in the injection molding process. However, the total crystallinity $\mathrm{X}_{\mathrm{c}}$ of sc-PLA (23.4\%) was much higher than that of PLLA because sc crystallites can act as nucleation sites to accelerate PLA crystallization rate significantly (Schmidt et al. 2001). By comparison, the two cellulose fibers/sc-PLA composites showed higher total $\left(\mathrm{X}_{\mathrm{c}}\right)$ and stereocomplex $\left(\mathrm{X}_{\mathrm{sc}}\right)$ crystallinities. The relative amount of sc crystallites $\left(f_{s c}\right)$ exceeded that of sc-PLA because the cellulose fibers can act as nucleating agents to further accelerate PLA crystallization (Masirek et al. 2007).

It is worth noting that Lyocell fibers were more effective than flax fibers in accelerating the crystallinity and formation of sc crystallites. This may be because Lyocell fibers have lower diameter, therefore there is greater surface area per weight of Lyocell fibers to serve as nucleation sites, promoting sc-PLA crystallinity.

\section{TG analysis}

Figure 5 shows TG curves of injection-molded PLLA, sc-PLA, and the cellulose fiber/sc-PLA composites. It can be noticed that injection-molded PLLA and scPLA showed the similar thermal stability, whereas the degradation temperatures of two kinds of cellulose fiber/sc-PLA composites were lower than those of scPLA or PLLA, which might be caused by the lower degradation temperature of cellulose vs. PLA. By comparison, the thermal degradation temperature of Lyocell/sc-PLA composite was lower than flax/scPLA composite, which was related with the thermal stabilities of fibers (Fig. 3).

\section{Mechanical properties}

Figures 6 and 7 show the tensile properties and Izod notched impact strengths, respectively, of injectionmolded PLLA, sc-PLA, and the cellulose fiber/sc-PLA composites. Tensile and notched impact strength

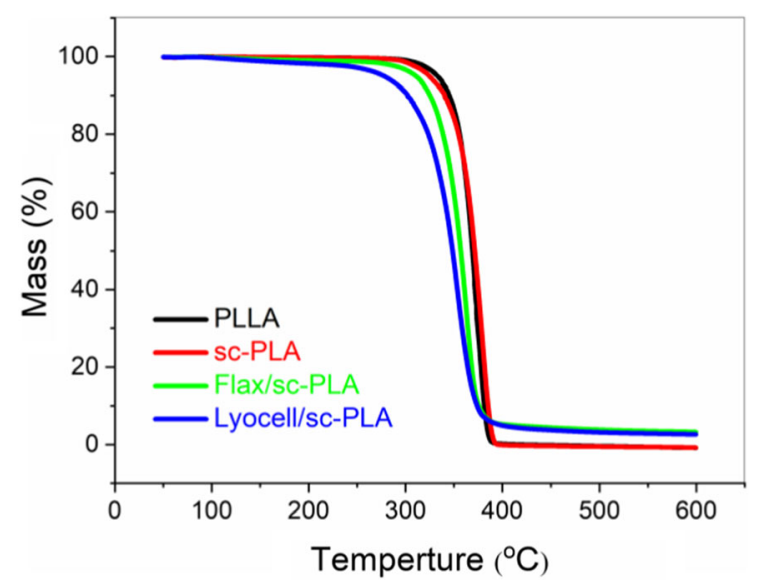

Fig. 5 TG curves of injection-molded PLLA, sc-PLA and different cellulose fiber/sc-PLA composites 
(a)

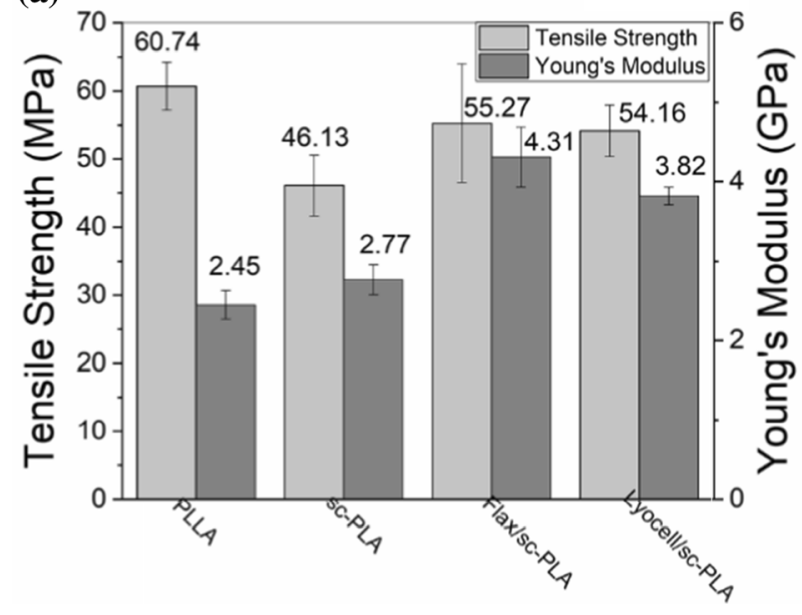

(b)

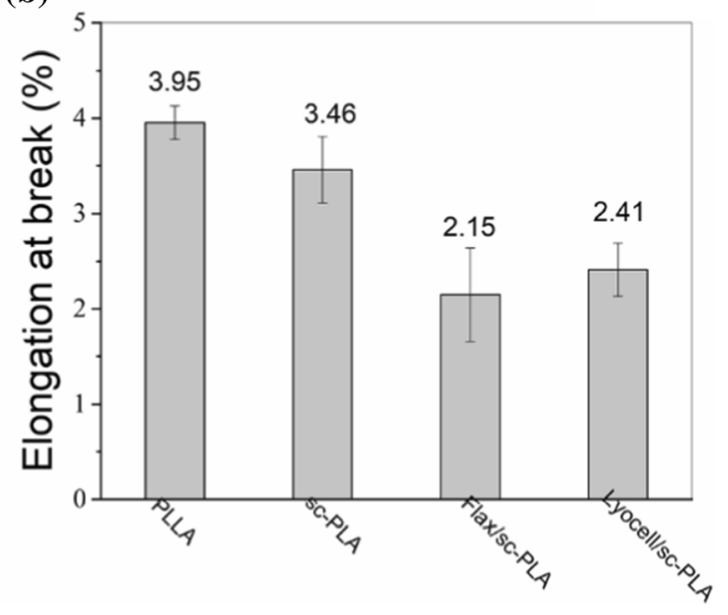

Fig. 6 Tensile properties of injection-molded PLLA, sc-PLA, and cellulose fiber/sc-PLA composites

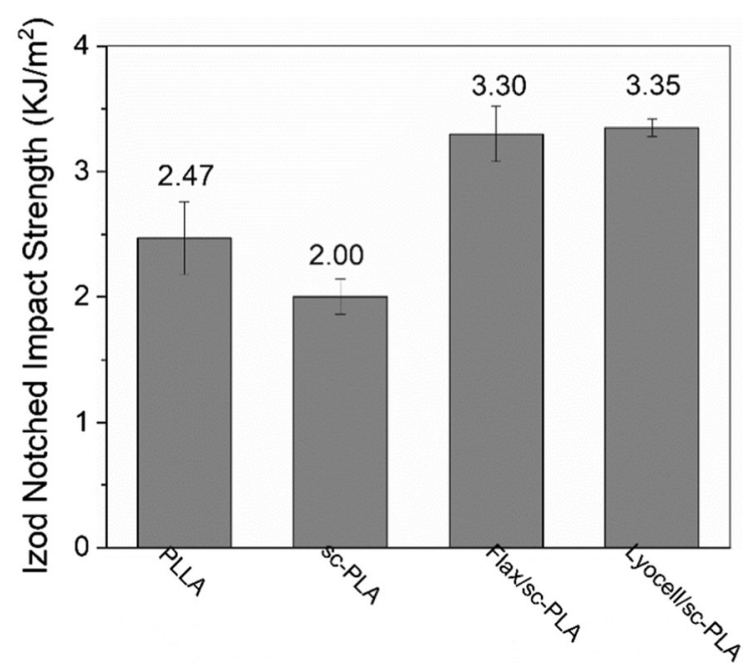

Fig. 7 Izod notched impact strengths of injection-molded PLLA, sc-PLA, and cellulose fiber/sc-PLA composites

values of injection-molded sc-PLA were $46.13 \mathrm{MPa}$ and $2.00 \mathrm{~kJ} / \mathrm{m}^{2}$, respectively, or $24.1 \%$ and $19.0 \%$ lower than the corresponding measured PLA properties. This is likely due to partial degradation of the PLA molecular chain caused by the high temperature $\left(220{ }^{\circ} \mathrm{C}\right)$ melt processing of sc-PLA, and the phase separation between sc and hc in sc-PLA (Brochu et al. 1995; Xu et al. 2012). However, sc-PLA showed improved Young's modulus, about $13.1 \%$ higher than that of PLA, and the elongation of sc-PLA decreased from $3.95 \%$ to $3.46 \%$, which may be due to the increased crystallinity of sc-PLA.
Mechanical properties of sc-PLA composites improved greatly when reinforced with either flax or Lyocell fibers. Although the mechanical properties of Lyocell fibers were inferior to those of flax fibers, both flax/sc-PLA and Lyocell/sc-PLA composites had similar tensile strength values, $19.8 \%$ and $17.4 \%$ higher, respectively, than those of sc-PLA. The possible reason is that flax may degrade at the higher processing temperature due to its lower thermal stability vs. Lyocell fibers, which decreased the reinforcing effect of flax in composites.

However, flax/sc-PLA composites still displayed higher Young's Modulus values than those of Lyocell/ sc-PLA composites due to excellent modulus of flax. The Young's moduli of flax/sc-PLA and Lyocell/scPLA composites were increased vs. sc-PLA by $55.6 \%$ and $37.9 \%$, respectively. The improved tensile strengths and Young's moduli of cellulose fiber/scPLA composites may be attributed to effective stress transfer from sc-PLA to the cellulose fibers, and the improved crystallinity of the composites. The higher elongation can be found for Lyocell/sc-PLA composites, compared with flax/sc-PLA composites, which may be related with the higher elongation of Lyocell fiber vs. flax.

Impact strength values of flax/sc-PLA and Lyocell/ sc-PLA composites were improved $65.0 \%$ and $67.5 \%$ vs. sc-PLA, respectively (Fig. 7), indicating that the non-satisfying impact strength of sc-PLA could be increased by reinforcing with cellulose fibers. Lyocell fibers are more effective in improving impact strength 
than are flax fibers, likely due to their higher elongation.

It is noteworthy that sc-PLA composites with natural flax fibers showed high variability in terms of mechanical properties, due to the non-uniformity of those natural fibers. By contrast, more stable mechanical properties with lower variability could be found for sc-PLA composites with Lyocell, which is spun from a solution of purified dissolving pulp in $\mathrm{N}$ methylmorpholine oxide and water.

\section{Water contact angle analysis}

Figure 8 shows water contact angles of injectionmolded PLLA, sc-PLA, and the cellulose fiber/sc-PLA composites. It's very obvious that PLLA had the contact angle of $104.79^{\circ}$, which confirmed the typical hydrophobicity of PLLA. Surprisingly, sc-PLA showed the weak hydrophobicity with the contact angle of $91.70^{\circ}$, which maybe related with the socalled $\beta$-form sc crystallites in sc-PLA (Brizzolara et al. 1996). Therefore, PLLA and sc-PLA exhibited different surface properties, which may have significant influence on the interfacial adhesion between cellulose fibers and PLA matrix.

Compared with sc-PLA, cellulose fiber/sc-PLA composites showed lower contact angles, which were resulted from the hydrophilic cellulose fibers. The higher contact angle of flax/sc-PLA composites (89.76 $\left.{ }^{\circ}\right)$ vs. flax/sc-PLA composites $\left(84.31^{\circ}\right)$, was mainly attributed to the hydrophobic phenylpropane structure of lignin in flax.

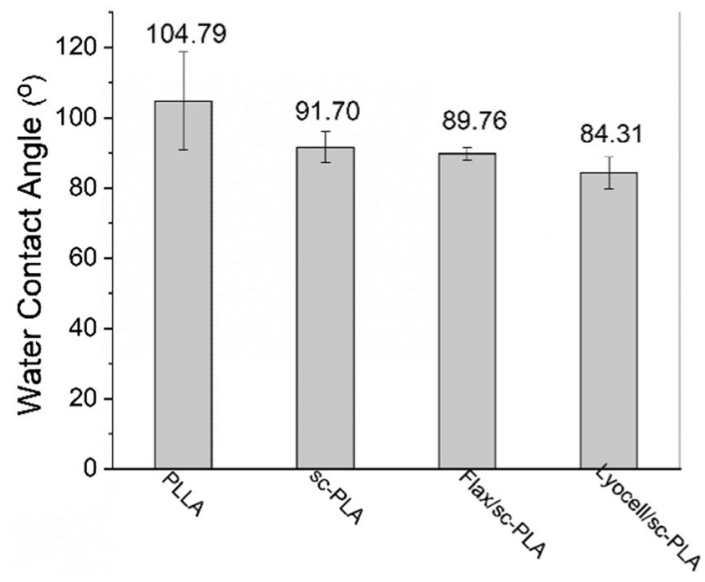

Fig. 8 Water contact angles of injection-molded PLLA, scPLA, and cellulose fiber/sc-PLA composites
SEM analysis

Figure 9 shows SEM images of fracture surfaces of injection-molded sc-PLA and the two types of cellulose fiber/sc-PLA composites. Compared with scPLA, a rough fracture interface can be seen for flax fiber/sc-PLA composite, the surfaces of pull-out flax fibers were covered with the matrix, and no gaps were visible between fiber and sc-PLA matrix, which suggests there is good adhesion between them. The natural fibers usually have poor adhesion to hydrophobic PLLA, whereas the difference in surface properties (water contact angle) of PLLA and sc-PLA maybe one reason for the good adhesion of flax/sc-PLA composites. In other words, less hydrophobic sc-PLA had better adhesion with flax, compared with PLLA. Moreover, interaction between the hydrophobic lignin of flax fibers with the hydrophobic PLA matrix may improve fiber-matrix adhesion (Graupner 2008). By contrast, there were obvious gaps between Lyocell fiber and matrix, and long and smooth fiber pull-outs could be observed in the fracture surface of Lyocell/ sc-PLA composite with several typical cylindrical voids, indicating limited adhesion between (essentially lignin-free) Lyocell fibers and the PLA matrix.

\section{VST Analysis}

Figure 10 shows the VST values of injection-molded PLLA, sc-PLA, and the cellulose fiber/sc-PLA composites. VST is an important parameter to evaluate the thermal properties of materials. The higher the VST, the higher the heat stability. It can be seen from the Fig. 10 that the VST of PLLA was the lowest, at only $60.0{ }^{\circ} \mathrm{C}$, and sc-PLA prepared by melt compounding and injection molding also exhibited a low VST of $69.1{ }^{\circ} \mathrm{C}$, which may be caused by the high content (35.7\%) of homocrystallites in sc-PLA and possible phase separation between stereocomplex crystallites and homocrystallites (Ming et al. 2017).

In contrast, the VST of sc-PLA composites improved significantly by reinforcing with flax or Lyocell fibers because fiber reinforcement can prevent deformation of the sc-PLA composite, and increase the stereocomplex crystallites content and crystallinity in the composites. The VST of flax/sc-PLA and Lyocell/sc-PLA composites can reach $159{ }^{\circ} \mathrm{C}$ and $170{ }^{\circ} \mathrm{C}$, respectively, about $100{ }^{\circ} \mathrm{C}$ higher than PLLA or sc-PLA, indicating that higher thermal stability can 

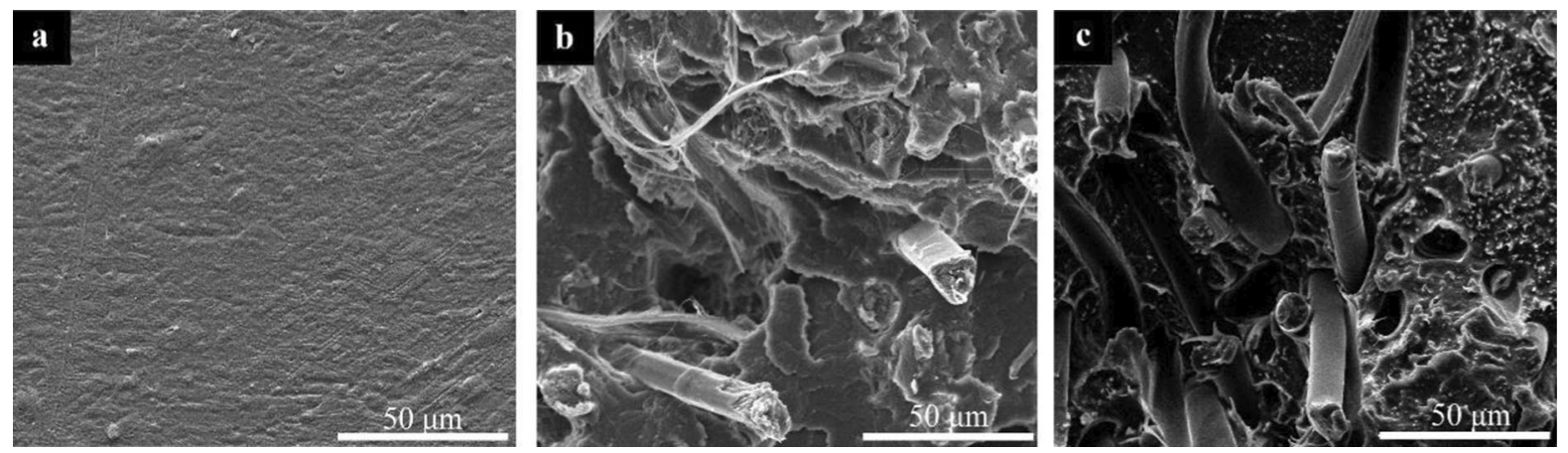

Fig. 9 SEM images of fracture surface of injection-molded sc-PLA (a), flax/sc-PLA (b) and Lyocell/sc-PLA composites (c)

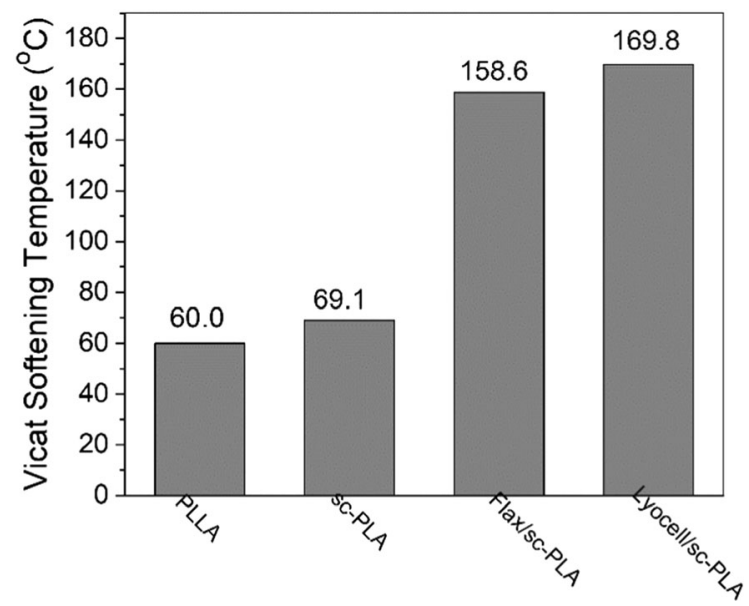

Fig. 10 Vicat softening temperatures of injection-molded PLLA, sc-PLA, and cellulose fiber/sc-PLA composites

be obtained by the addition of flax and Lyocell fibers. Compared with the flax/sc-PLA composite, the Lyocell/sc-PLA composite exhibited higher VST due to its higher crystallinity and content of stereocomplex crystallites (Table 3).

\section{Conclusions}

A natural cellulose fiber, flax, and a regenerated fiber spun from cellulose dissolving pulp, Lyocell, were used to prepare cellulose fibers/sc-PLA composites. The two cellulose-based fibers differ in important properties that result from their divergent source and preparation methods, including composition, shape, and surface roughness. These property differences impact composite performance. We found that thermal stability and mechanical properties of PLA could both be improved by stereocomplex formation and by reinforcement with cellulose fibers. These findings will expand the potential applications of PLA-based materials. The mechanical property of a reinforcing fiber is not the single and decisive factor for the properties of composites. Higher purity, elongation, or degree of roughness may have significant impact upon mechanical properties and thermal resistance. Mechanical properties of dissolved and extruded, low-hemicellulose Lyocell fibers were significantly weaker than those of natural flax fibers. In contrast, Lyocell/sc-PLA composites showed higher impact strength and similar tensile strength versus flax/sc-PLA composites, and Lyocell fibers were more effective in accelerating crystallinity and formation of sc crystallites than flax fibers. As a result, the highest Vicat softening temperature of $170{ }^{\circ} \mathrm{C}$ was observed for Lyocell/sc-PLA composites. Therefore, Lyocell fiber may be especially promising in the field of sustainable-based, reinforced composites due to its good reinforcing ability, especially its lower variability vs. flax fibers.

Funding Financial supports from National Key Research and Development Program of China (2017YFB0309501) and Natural Science Foundation of Shanghai (16ZR1401600) are gratefully acknowledged.

\section{Declarations}

Conflict of interest The authors declare that there is no conflict of interest, and no animal studies or human participants were involved in this study.

\section{References}

Arao Y, Fujiura T, Itani S, Tanaka T (2015) Strength improvement in injection-molded jute-fiber-reinforced 
polylactide green-composites. Compos Part B-Eng 68:200-206

Aydin M, Tozlu H, Kemaloglu S, Aytac A, Ozkoc G (2011) Effects of alkali treatment on the properties of short flax fiber-poly(lactic acid) eco-composites. J Polym Environ 19:11-17

Bax B, Müssig J (2008) Impact and tensile properties of PLA/ Cordenka and PLA/flax composites. Compos Sci Technol 68:1601-1607

Biagiotti J, Puglia D, Torre L, Kenny LT, Arbelaiz A, Cantero G, Marieta C, Llano-Ponte R, Mondragon I (2004) A systematic investigation on the influence of the chemical treatment of natural fibers on the properties of their polymer matrix composites. Polym Composite 25:470-479

Brizzolara D, Cantow HJ, Diederichs K, Keller E, Domb AJ (1996) Mechanism of the stereocomplex formation between enantiomeric poly(lactide)s. Macromolecules 29:191-197

Brochu S, Prud'homme RE, Barakat I, Jérôme R, (1995) Stereocomplexation and morphology of polylactides macromolecules. Macromolecules 28:5230-5239

Ganster J, Fink H-P (2006) Novel cellulose fibre reinforced thermoplastic materials. Cellulose 13:271-280

Ghosh S, Viana JC, Reis RL, Mano JF (2007) Effect of processing conditions on morphology and mechanical properties of injection-molded poly(l-lactic acid). Polym Eng Sci 47:1141-1147

Graupner N (2008) Application of lignin as natural adhesion promoter in cotton fibre-reinforced poly(lactic acid) (PLA) composites. J Mater Sci 43:5222-5229

Graupner N, Herrmann AS, Müssig J (2009) Natural and manmade cellulose fibre-reinforced poly(lactic acid) (PLA) composites: an overview about mechanical characteristics and application areas. Compos Part A-Appl S 40:810-821

Graupner N, Rößler J, Ziegmann G, Müssig J (2014) Fibre/matrix adhesion of cellulose fibres in PLA, PP and MAPP: a critical review of pull-out test, microbond test and single fibre fragmentation test results. Compos Part A-Appl S 63:133-148

Hu R, Lim JK (2007) Fabrication and mechanical properties of completely biodegradable hemp fiber reinforced polylactic acid composites. J Compos Mater 41:1655-1669

Huda MS, Drzal LT, Misra M, Mohanty AK (2006) Wood-fiberreinforced poly(lactic acid) composites: evaluation of the physicomechanical and morphological properties. J Appl Polym Sci 102:4856-4869

Huda MS, Drzal LT, Mohanty AK, Misra M (2008) Effect of fiber surface-treatments on the properties of laminated biocomposites from poly(lactic acid) (PLA) and kenaf fibers. Compos Sci Technol 68:424-432

Ikada Y, Jamshidi K, Tsuji H, Hyon SH (1987) Stereocomplex formation between enantiomeric poly(lactides). Macromolecules 20:904-906

Jiang X, Bai Y, Chen X, Liu W (2020) A review on raw materials, commercial production and properties of lyocell fiber. J Bioresour Bioprod 5:17-27

Jin F-L, Hu R-R, Park S-J (2019) Improvement of thermal behaviors of biodegradable poly(lactic acid) polymer: a review. Compos Part B-Eng 164:287-296

Li Y, Li Q, Yang G, Ming R, Yu M, Zhang H, Shao H (2018) Evaluation of thermal resistance and mechanical properties of injected molded stereocomplex of poly(l-lactic acid) and poly(d-lactic acid) with various molecular weights. Adv Polym Tech 37:1674-1681

Liu H, Zhang J (2011) Research progress in toughening modification of poly(lactic acid). J Polym Sci Pol Phys 49:1051-1083

Ma H, Joo CW (2011) Structure and mechanical properties of jute-polylactic acid biodegradable composites. J Compos Mater 45:1451-1460

Masirek R, Kulinski Z, Chionna D, Piorkowska E, Pracella M (2007) Composites of poly(L-actide) with hemp fibers: morphology and thermal and mechanical properties. J Appl Polym Sci 105:255-268

Mathew AP, Oksman K, Sain M (2006) The effect of morphology and chemical characteristics of cellulose reinforcements on the crystallinity of polylactic acid. J Appl Polym Sci 101:300-310

Ming R, Yang G, Li Y, Wang R, Zhang H, Shao H (2017) Flax fiber-reinforced polylactide stereocomplex composites with enhanced heat resistance and mechanical properties. Polym Composite 38:472-478

Mokhena TC, Sefadi JS, Sadiku ER, John MJ, Mochane MJ, Mtibe A (2018) Thermoplastic processing of PLA/cellulose nanomaterials composites. Polymers 10:1363

Mwaikambo LY, Ansell MP (2002) Chemical modification of hemp, sisal, jute, and kapok fibers by alkalization. J Appl Polym Sci 84:2222-2234

Nassiopoulos E, Njuguna J (2015) Thermo-mechanical performance of poly(lactic acid)/flax fibre-reinforced biocomposites. Mater Design 66:473-485

Ochi S (2008) Mechanical properties of kenaf fibers and kenaf/ PLA composites. Mech Mater 40:446-452

Orue A, Jauregi A, Peña-Rodriguez C, Labidi J, Eceiza A, Arbelaiz A (2015) The effect of surface modifications on sisal fiber properties and sisal/poly (lactic acid) interface adhesion. Compos Part B-Eng 73:132-138

Río JCd, Gutiérrez A, Rodríguez IM, Ibarra D, Martínez áT, (2007) Composition of non-woody plant lignins and cinnamic acids by Py-GC/MS, Py/TMAH and FT-IR. J Anal Appl Pyrol 79:39-46

Sarasua JR, Arraiza ALp, Balerdi P, Maiza I, (2005) Crystallinity and mechanical properties of optically pure polylactides and their blends. Polym Eng Sci 45:745-753

Sawpan MA, Pickering KL, Fernyhough A (2011) Improvement of mechanical performance of industrial hemp fibre reinforced polylactide biocomposites. Compos Part A-Appl S 42:310-319

Schmidt SC, Hillmyer MA (2001) Polylactide stereocomplex crystallites as nucleating agents for isotactic polylactide. J Polym Sci Pol Phys 39:300-313

Sgriccia N, Hawley MC, Misra M (2008) Characterization of natural fiber surfaces and natural fiber composites. Compos Part A-Appl S 39:1632-1637

Srithep Y, Pholharn D, Turng L-S, Veang-in O (2015) Injection molding and characterization of polylactide stereocomplex. Polym Degrad Stabil 120:290-299

Tokoro R, Vu DM, Okubo K, Tanaka T, Fujii T, Fujiura T (2007) How to improve mechanical properties of polylactic acid with bamboo fibers. J Mater Sci 43:775-787 
Tsuji H, Ikada Y (1993) Stereocomplex formation between enantiomeric poly(lactic acids). 9 Stereocomplexation from the Melt. Macromolecules 26:6918-6926

Tsuji H, Ikada Y (1999) Stereocomplex formation between enantiomeric poly(lactic acid)s. XI. mechanical properties and morphology of solution-cast films. Polymer 40:6699-6708

Xu H, Tang S, Chen J, Yin P, Pu W, Lu Y (2012) Thermal and phase-separation behavior of injection-molded poly(l-lactic acid)/poly(d-lactic acid) blends with moderate optical purity. Polym Bull 68:1135-1151

Yang H, Rong Y, Chen H, Dong HL, Zheng C (2007) Characteristics of hemicellulose, cellulose and lignin pyrolysis. Fuel 86:1781-1788

Yu M, Zhang H, Liu Z, Ge Z, Kong F, Shao H, Hu X (2019) Effects of fiber dimension and its distribution on the properties of Lyocell and ramie fibers reinforced polylactide composites. Fiber Polym 20:1726-1732
Zhang J, Sato H, Tsuji H, Noda I, Ozaki Y (2005) Differences in the $\mathrm{CH}_{3} \mathrm{O}=\mathrm{C}$ interactions among poly(L-lactide), poly(Llactide)/poly(D-lactide) stereocomplex, and poly(3-hydroxybutyrate) studied by infrared spectroscopy. J Mol Struct 735-736:249-257

Zhang H, Ming R, Yang G, Li Y, Li Q, Shao H (2015) Influence of alkali treatment on flax fiber for use as reinforcements in polylactide stereocomplex composites. Polym Eng Sci 55:2553-2558

Zhang H, Li Y, Yang G, Yu M, Shao H (2020) Effect of interfacial modification on the thermo-mechanical properties of flax reinforced polylactide stereocomplex composites. J Polym Eng 40:403-408

Publisher's Note Springer Nature remains neutral with regard to jurisdictional claims in published maps and institutional affiliations. 